\title{
Precision Mapping of Apple Proliferation using Multi- and Hyperspectral Data
}

\author{
Ben Alexander McLeod ${ }^{\text {a,c }}$, Ekaterina Chuprikova ${ }^{a}$, Abraham Mejia-Aguilar ${ }^{\text {a }}$, Dana Barthel ${ }^{\text {b }}$ \\ Katrin Janik ${ }^{\mathrm{b}}$, Ulrich Prechsl ${ }^{\mathrm{b}}$, Roberto Monsorno ${ }^{\mathrm{a}}$, Liqiu Meng ${ }^{\mathrm{c}}$ \\ ${ }^{a}$ Eurac Research, Center for Sensing Solutions, Ekaterina.Chuprikova@eurac.edu., Abraham.Mejia@eurac.edu, \\ Roberto.Monsorno@eurac.edu \\ ${ }^{b}$ Laimburg Research Centre, Department for Functional Genomics, Dana.Barthel@laimburg.it, Katrin.Janik@laimburg.it, \\ Ulrich.Prechsl@laimburg.it \\ ${ }^{c}$ Technical University Munich, Department for Aerospace and Geodesy, Chair of Cartography, Liqiu.Meng@tum.de \\ * Corresponding author,bmcleod@posteo.net
}

Keywords: Sensor-Driven Mapping, Plant Disease Mapping, Precision Map, Hyperspectral Data, Agriculture Mapping

Abstract:

This research aims at developing a cartographic understanding and methodology for producing precision maps to support local, tech-minded farmers, scientists, and authorities in making spatial decisions. Despite being a widely used cartographic product, the precision map contemporarily exists outside of a theoretical cartographic framework. Geographical Information Science and Remote Sensing on the other hand have already found entry in agricultural practices (Usery, Pocknee, \& Boydell, 1995; Papp et al., 2021), laying the foundation for precision agriculture (Sishodia et al., 2020). Making use of sensing systems to obtain (near real-time) environmental data, precision agriculture relies on the extraction of relevant, high-resolution geoinformation to be communicated visually to the end-user (Sishodia et al., 2020). Cartography as an interdisciplinary domain bears a huge potential in meeting the increasing demand for the decision-supporting visualisation of Big Data (Coetzee et al., 2020; Robinson et al., 2017), and thus may contribute to the development of more sustainable agriculture.

The focus of this research is on plant disease detection, as plant pests endanger tree health and harvest, thus highlighting farmers' vulnerability to plant diseases and ultimately undermining Sustainable Development Goals, such as Zero Hunger and food security (Savary et al., 2012). In contrast to traditional plant disease management, plant health monitoring through hyperspectral data would enable stakeholders to detect plant stress outside the range of visible light, and hence, at an early disease stage. Particularly, near-and short-wave infrared bands provide crucial information on plant health, allowing early intervention in plant disease control (Lowe, Harrison, \& French, 2017). Apple Proliferation (AP), a phytoplasma disease transmitted through insects, will be used as a case study in the Upper Adige Valley in South Tyrol (Italy) to propose a technology-and data-driven strategy for an early, non-destructive plant disease detection and monitoring based on hyperspectral data.

The work in progress consists of the following research objectives:

- developing a cartographic understanding and definition for a precision map,

- identifying relevant spectral bands (SB's) and vegetation indices (VI's) that allow meaningful discrimination between leaves infected with AP and uninfected leaves (binary discrimination),

- $\quad$ implementing a robust and reproducible image classification procedure based on a machine learning approach specifically for the identification of AP on varying levels of detail (at the leaf, tree, and orchard level),

- $\quad$ establishing a mapping technique to produce precision maps dedicated to AP on different levels of detail

Following a multi-modal and multi-scale data acquisition approach, several remote sensing techniques were applied: adopting a ground sensing approach, individual leaf spectral signatures were obtained through a spectroradiometer equipped with a leaf clipping tool extension; airborne proximal sensing was conducted on the orchard level using UAVmounted multi-and hyperspectral cameras. Insights gained through the spectral data analysis served as the basis for the subsequent multi- and hyperspectral waveband selection and composition of adequate vegetation indices. Additionally, machine learning techniques were adopted for image classification to delineate infected from uninfected trees. A hexagonal grid structure assimilating the tree rows is used for cartographic generalisation of the image classifications as well as for abstraction of the tree canopy shape by the individual hexagonal cell. Finally, the high-resolution classification on the leaf level and the aggregated spatial information regarding the AP infection on the tree and orchard level (i.e. as gridded abstraction) are presented as interrelated map-layers. 
Following Shneiderman's (1996) visualisation mantra Overview first, zoom and filter, then details-on-demand, the map user will first be presented with a general overview of aggregated spatial data on the orchard level, from which he/she can move between layers through zooming to obtain further detail at the tree and intra-tree level. This multi-scale view in precision mapping is also temporal in nature, as multiple UAV flights were undertaken in several phenological stages, providing further insight into the spatio-temporal disease evolution of Apple Proliferation.

The findings will be available on the Eurac Research Environmental Data Platform (https://www.maps.eurac.edu), a geoportal based on an open spatial data infrastructure (SDI) and managed by the Center for Sensing Solutions. Internally referred to as Maps, the geoportal is based on software maintained by the Open Source Geospatial Foundation (OSGeo), such that the interactive map user interface is based on the framework of geoNode and OpenLayers. The spatial data is managed through PostGIS (a PostgreSQL extension), which is then hosted through GeoServer that in turn is coupled with pycsw as well as GeoNetwork as respective metadata catalogues. Following Coetzee et al. 's idea (2020), this research is anticipated to be open science, thus findings and geodata from this research should be accessible to the public through FAIR (findable, accessible, interoperable, reusable) (Wilkinson et al. 2016), free and open-source geospatial software and infrastructure as well as map services. This experimental case study is expected to encourage future development and implementation of cartography in agriculture and foster the cartographic understanding and application of precision mapping using multi- and hyperspectral data.

This research is partially funded by the ERASMUS+ Student Mobility Program (Scholarship) and supported by the European Union's Horizon 2020 research and innovation program under the Marie Skłodowska-Curie grant agreement No 894215.

\section{References:}

Coetzee, S., Ivánová, I., Mitasova, H., \& Brovelli, M. (2020). Open Geospatial Software and Data: A Review of the Current State and A Perspective into the Future. ISPRS International Journal of Geo-Information, 9(2), 90.

Lowe, A., Harrison, N., \& French, A. P. (2017). Hyperspectral image analysis techniques for the detection and classification of the early onset of plant disease and stress. Plant Methods, 13(1), 1-12.

Papp, L., van Leeuwen, B., Szilassi, P., Tobak, Z., Szatmári, J., Árvai, M., Mészáros, J., \& Pásztor, L. (2021). Monitoring Invasive plant species using hyperspectral remote sensing data. Land, 10(1), 1-18.

Robinson, A. C., Demšar, U., Moore, A. B., Buckley, A., Jiang, B., Field, K., Kraak, M.-J., Camboim, S.P., \& Sluter, C. R. (2017). Geospatial big data and cartography: research challenges and opportunities for making maps that matter. International Journal of Cartography, 3(sup1), 32-60.

Savary, S., Ficke, A., Aubertot, J. N., \& Hollier, C. (2012). Crop losses due to diseases and their implications for global food production losses and food security. Food Security, 4(4), 519-537.

Shneiderman, B. (1996). The eyes have it: a task by data type taxonomy for information visualizations. Proceedings 1996 IEEE Symposium on Visual Languages, 336-343.

Sishodia, R. P., Ray, R. L., \& Singh, S. K. (2020). Applications of remote sensing in precision agriculture: A review. Remote Sensing, 12(19), 1-31.

Usery, E. L., Pocknee, S., \& Boydell, B. (1995). Precision farming data management using geographic information systems. Photogrammetric Engineering \& Remote Sensing, 61(11), 1383-1391.

Wilkinson, M. D. et al. (2016). The FAIR Guiding Principles for scientific data management and stewardship. Scientific Data, 3(1). 